\title{
Alpers syndrome presenting with anatomopathological features of fulminant autoimmune hepatitis
}

\author{
Peter Witters • Jacques Pirenne • Raymond Aerts • Diethard Monbaliu • \\ Frederik Nevens • Chris Verslype • Wim Laleman • Tania Roskams • Lars Desmet • \\ Dirk Vlasselaers • Paul Mariën • Ilse Hoffman • Rita Lombaerts • Eveline Goethals • \\ Jaak Jaeken • Wouter Meersseman • David Cassiman
}

Received: 22 January 2010 /Revised: 31 March 2010 /Accepted: 12 April 2010 /Published online: 26 June 2010

(C) SSIEM and Springer 2010

A 6-month-old girl with normal psychomotor development presented with fulminant hepatitis. Interestingly, liver biopsy demonstrated features of autoimmune hepatitis, whereas autoantibodies and raised immunoglobulin $\mathrm{G}(\mathrm{IgG})$ were absent. There was extensive centrolobular necrosis with bridging (Fig. 1a) and ductular reaction (Fig. 1b). There were numerous lymphocytes and plasmocytes (polyclonal on kappa and lambda stain) (Fig. 1c). Biochemical investigations excluded alternative causes of fulminant hepatitis. Steroid treatment was started, but the patient soon evolved to liver failure and received a liver transplantation.

Three months posttransplant, the patient developed myoclonic and generalized tonic-clonic fits. Computed tomography $(\mathrm{CT})$ scan, normal at presentation, revealed evolution toward severe brain atrophy. Mitochondrial assays on postmortem muscle biopsy revealed normal activity of respiratory chain complex II and decreased I, III, and IV activity. This is indicative of mitochondrial DNA (mtDNA) depletion in this clinical setting (Hargreaves et al. 2002). DNA analysis revealed two known POLG mutations (p.Ala467Thr and p. Gly848Ser), diagnostic for Alpers syndrome.

We show here for the first time that a mtDNA-depletion syndrome can present with the anatomopathological features of autoimmune hepatitis. Alpers syndrome therefore should not be excluded from the differential diagnostic list

Communicated by: Shamima Rahman

Competing interest: None declared.

P. Witters $(\triangle) \cdot$ J. Pirenne $\cdot$ R. Aerts $\cdot$ D. Monbaliu $\cdot$ F. Nevens $\cdot$

C. Verslype $\cdot$ W. Laleman $\cdot$ T. Roskams $\cdot$ L. Desmet

D. Vlasselaers · P. Mariën · I. Hoffman · R. Lombaerts •

E. Goethals $\cdot$ J. Jaeken $\cdot$ W. Meersseman $\cdot$ D. Cassiman

University Hospital Gasthuisberg, KU Leuven,

Leuven, Belgium

e-mail: peter.witters@gmail.com

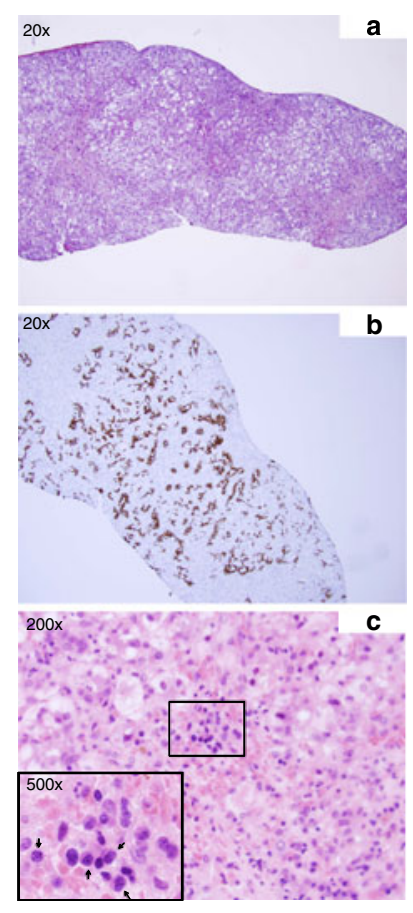

Fig. 1 a Sirius red stain. b CK7 stain. c Hematoxylin-eosin stain: arrows indicate plasmocytes

based on these features. Whether this is also the case in older children, or even adults, presenting with cryptogenic acute liver failure and pathological features of autoimmune hepatitis remains to be determined.

\section{Reference}

Hargreaves P, Rahman S, Guthrie P et al (2002) Diagnostic value of succinate ubiquinone reductase activity in the identification of patients with mitochondrial DNA depletion. J Inherit Metab Dis 25:7-16 